\title{
Aportes instrumentales y sustantivos de Blockchain a la educación
}

\section{Instrumental and substantial contributions of Blockchain to education}

\author{
Santiago Tomás Bellomo \\ e-mail: sbellomo@austral.edu.ar \\ Universidad Austral. Argentina
}

\begin{abstract}
Resumen
Blockchain irrumpe en el escenario educativo ofreciendo alternativas de mejora sobre ciertos procesos administrativos, como son el reconocimiento de identidad del usuario, la validación no mediada de certificados académicos, o la utilización de contratos inteligentes, entre otras novedades. Ciertas crónicas futuristas auguran que — gracias a Blockchain — se desarrollará una transformación de la arquitectura del sistema educativo en su conjunto y una sinergia sin precedentes entre docentes, instituciones y ámbitos formales y no formales de educación. Si bien los avances son incipientes y los trabajos académicos escasos, existen indicios claros sobre la potencialidad de Blockchain en su aplicación administrativa, y resultan interesantes (a la par que desafiantes) algunas iniciativas educativas basadas en el modelo de las plataformas tecnológicas multilaterales. Es preciso advertir, sin embargo, sobre el escaso aporte que conlleva la aplicación de tecnología en la dimensión pedagógica, y sobre la incertidumbre que se cierne sobre las pretensiones revolucionarias de las prospectivas más extremas, que pronostican más caudal de transformación que el que la tecnología pareciera ofrecer.
\end{abstract}

Palabras clave: Blockchain; certificación; acreditación; tecnología educativa; contratos inteligentes.

\section{Abstract}

Blockchain breaks into the educational scenario offering alternatives for improvement over certain administrative processes, such as recognition of user identity, non-mediated validation of academic certificates, or the use of smart contracts, among other novelties. Futuristic chronicles predict a transformation of the architecture of the educational system as a whole and an unprecedented synergy between teachers, institutions and formal and non-formal areas of education. Although the advances are in their infancy and there isn't much academic research on the subject yet, it is clear that Blockchain has great potential with regards to its administrative application. Some educational initiatives based on the model of multilateral technological platforms result to be interesting as well as challenging. It is necessary to warn, however, about the little contribution that the application of Blockchain technology entails in the pedagogical dimension, and about the uncertainty that hangs over the revolutionary claims of the most extreme prospects, which predict more transformative power than what the technology itself seems to offer.

Keywords: Blockchain; certification; accreditation; ed-tech; smart contracts.

Recibido / Received: 28-01-2019

Aceptado / Accepted: 02-09-2019

Publicación en linea / Published online: 20-12-2019

Cómo referenciar este artículo / How to reference this article:

Bellomo, S. (2020). Aportes instrumentales y sustantivos de Blockchain a la educación. Tendencias Pedagógicas, 35, pp. 34-48. doi: 10.15366/tp2020.35.004 


\section{Introducción}

Blockchain es una base de datos distribuida entre todas las computadoras que forman parte de una red. A diferencia de las redes tradicionales, es pública (todo el mundo puede verla cuando quiera), distribuida (se ejecuta en múltiples ordenadores) y encriptada (incluye claves públicas y privadas que garantizan su seguridad).

El surgimiento del Blockchain ha actualizado y profundizado algunos debates relativos al sistema educativo, tanto en lo concerniente a aspectos instrumentales (sistemas de pago o trazabilidad de certificaciones) como en cuanto a aspectos determinantes de su arquitectura actual y el modo en que resuelve la enseñanza personalizada o personalización (King, Prince \& Swanson, 2016). Las voces más entusiastas declaran que Blockchain revolucionará la educación tal como la conocemos (Vander Ark, 2018b; Chen, Xu, Lu, \& Chen, 2018; Clark, 2016). ¿Qué hay de cierto en ello?

El alto grado de receptividad que viene teniendo esta tecnología en la opinión educativa se explica, entre otras razones, en función del contexto más amplio de demandas que se vuelcan sobre la educación contemporánea. El necesario «aprendizaje para toda la vida» preanunciado por Delors en 1996 cobra mayor relevancia en la medida en que florece la ubicuidad del aprendizaje. Ella permite al alumno romper la dependencia radical de las variables tiempo y espacio que acompañan a la educación presencial y multiplica exponencialmente las posibilidades de acceso a un sinnúmero de recursos en modalidad sincrónica y asincrónica, a partir de sus atributos sobreañadidos: portabilidad, interconexión, interactividad e inmersividad (Burbules, 2014, 16ss).

A la par de la transformación en la educación formal, florecen las herramientas complementarias para la capacitación en ámbitos no formales (Franceschin, 2017, 1): boot camps intensivos o nanotítulos (nanodegrees) en formato MOOC, típicos de plataformas como EDX o Coursera, así como comunidades de aprendizaje o bibliotecas de tutoriales como Academia Khan o YouTube, prometen complementar las competencias y habilidades de las personas y presentan el desafío de superar «la distinción entre educación formal — y por ende con una acreditación regulada — por un lado, y la educación no formal e informal —ambas carentes de acreditación, por otro» (Bartolomé, Bellver, Castañeda, \& Adell, 2017).

La factibilidad técnica de una mayor personalización del aprendizaje acrecienta la demanda de democratización del sistema educativo, y la habilitación de itinerarios de aprendizaje más ajustados a las posibilidades y necesidades individuales (Bartolomé et al, 2017). En este contexto, Blockchain se presenta como un jugador dispuesto a cambiar las reglas de juego del sistema.

En este trabajo se analizan los alcances de la transformación que surgen de la aplicación de Blockchain en educación. Tratándose de un proceso que aún está en su infancia (Grech \& Camilleri, 2017), cualquier juicio taxativo puede resultar apresurado. La naturaleza eminentemente técnica del tema expone, además, a la imprecisión o al error, mucho más cuando la materia analizada tiene que ver con aplicaciones futuras del Blockchain que aún nos resultan desconocidas.

En efecto, las fuentes principales de este trabajo no refieren solo a los progresos actuales específicos, sino también a los argumentos y consideraciones que nutren los documentos declarativos. Estos documentos reúnen «libros blancos» (white papers) fundacionales, artículos de divulgación científica o pedagógica, informes de organismos internacionales, prospectivas de carácter hipotético y unos pocos artículos académicos.

Por lo general, se trata de documentos que acompañan el surgimiento de iniciativas exploratorias que aplican Blockchain en algunas dimensiones de la actividad educativa, todavía en estado embrionario. La constitución argumentativa de varios de estos trabajos es naturalmente sesgada a favor de la incorporación de Blockchain, lo que no minimiza la eventual fuerza persuasiva de sus contenidos.

El análisis del fenómeno desde una perspectiva educativa destaca, por un lado, la potencialidad de la aplicación de Blockchain en aquellas dimensiones en las que la utilización de mecanismos descentralizados de verificación algorítmica resultaría sumamente atractiva y conveniente. Nos referimos a la verificación de identidad y la emisión, validación, conservación y compartición de certificados. La factibilidad de una aplicación más extensiva queda relativizada, en tanto y en cuanto las dimensiones más sustantivas de la educación (enseñanza o evaluación, por ejemplo) se resisten a ser reducidas según el criterio de la certificación algorítmica. El análisis revelará el fuerte talante racionalista de algunas prospectivas que proponen resolver vía Blockchain procesos semejantes.

En defensa de la personalización y la democratización, Blockchain reaviva el debate en torno al rol que cabe asignar a las instituciones formales, y al lugar que debe ocupar la educación no formal o 
la informal en el trayecto formativo de una persona. Asimismo, despierta interrogantes sobre el papel de los organismos públicos tanto en lo relativo a la acreditación de instituciones como a la determinación de estándares, criterios y contenidos mínimos propios del sistema educativo.

Finalmente, la instrumentación de proyectos educativos basados en Blockchain de código cerrado alerta sobre la posibilidad cierta de que se reemplace la actual dinámica de organización del sistema educativo por iniciativas particulares que puedan atentar contra la calidad, o generen nuevos tipos de dependencia contrarios al ideal de democratización y personalización que se pretende lograr.

\section{Qué es Blockchain}

El surgimiento del Blockchain data de 2008. Su invención se atribuye al pseudónimo de Satoshi Nakamoto $^{1}$, de quién no se sabe a ciencia cierta si es varón o mujer, o incluso si se trata de un individuo o grupo de individuos. Su trabajo original, así como el registro de implementación publicado al año siguiente, constituyen la piedra angular bajo la cual se construyó el sistema de criptomonedas Bitcoin.

Los expertos coinciden en señalar que la tecnología Blockchain tiene la potencialidad de transformar la actual Internet del intercambio de información en una nueva Internet del intercambio de valor (Chen et al, 2018). Blockchain atravesó exitosamente la etapa 1.0 (desarrollo de criptomonedas como un sistema de pagos peer-to-peer) y se encuentra en la 2.0 (que se extiende a otras áreas que mantienen este sistema de intercambio simple). Blockchain 3.0 está ensayando aplicaciones exploratorias en otras áreas de mayor complejidad, como el gobierno, la salud, la literatura, la ciencia, la cultura, la educación y el arte (Chen et al, 2018). En este último estadio, debe considerarse que nos encontramos ante un entorno ciertamente incipiente y salvaje, de un cierto Wild West del Blockchain, aunque se la considere una barbarie en cierta forma deseada, precisamente por su naturaleza exploratoria (Grech et al, 2017).

Son muchas las fuentes que ofrecen una noción básica acerca de Blockchain y sus particularidades tecnológicas (Grech et al, 2017; Chen et al, 2018). Suele indicarse que se trata de un libro de contabilidad público y digital, descentralizado y confiable; una red distribuida de computadoras (nodos) utilizadas para mantener la fuente de información compartida. Cada nodo garantiza la seguridad y calidad de la información al conservar una copia completa de los registros de transacciones pasadas. Así, a través de Blockchain, dos usuarios pueden realizar una determinada transacción, por ejemplo, de criptomonedas. En tanto la información de la transacción tiene un dominio privado y uno público, mantienen privacidad, pero un tercer usuario (llamado minero), a través de una clave pública, puede certificar la transacción resolviendo un acertijo matemático denominado proof-of-work2. Una vez validada la transacción mediante este protocolo de consenso, se crea un bloque que se añade a la cadena de transacciones (o bloques) que le antecede. Cada bloque contiene la información de la cadena completa, y la información está distribuida en todos los usuarios, por lo que no hay manera de alterar el registro.

Blockchain ofrece, por tanto, las siguientes características, que suelen ser vistas como ventajas comparativas respecto de otras tecnologías (Grech et al, 2017):

- La descentralización: el almacenamiento, mantenimiento, transmisión y verificación de la información está distribuida en un sistema no centralizado.

- La confianza entre los nodos distribuidos se construye a partir de parámetros matemáticos, por lo que no hace falta apelar a terceras organizaciones para que actúen como fuente de validación (bancos, escribanías, etc.)

\footnotetext{
${ }^{1}$ El trabajo de Nakamoto titulado Bitcoin: A Peer-to-Peer Electronic Cash System fue publicado el 31 de octubre de 2008 y describe el protocolo de la red Bitcoin y su arquitectura distribuida.

${ }^{2}$ En los párrafos siguientes se señalarán algunas derivaciones negativas asociadas con esta modalidad de consenso, y los actuales ensayos de otra modalidad de consenso o validación denominada Proof of Stake, que también ofrece sus virtudes y defectos.
} 
- La confiabilidad del dato y del sistema, a su vez, descansan en subpropiedades específicas: la trazabilidad, por un lado, que guarda relación con la posibilidad de reconocer y registrar el recorrido cronológico exacto de la cadena de bloques; la inmutabilidad de la información, por otro lado, que queda garantizada mediante mecanismos que impiden que un usuario altere información sin que los restantes nodos lo detecten; la fiabilidad del sistema, en tanto no hay dependencia prioritaria de un nodo centralizado específico, lo que reduce la exposición y vulnerabilidad al mínimo ante la imposibilidad de que todos los nodos sean anulados simultáneamente.

- Todas estas propiedades redundan en una mayor eficiencia, en la medida que la información fluye automáticamente, ajustada a procedimientos predeterminados, lo que supone una reducción de costos de mano de obra y elimina la posibilidad de error.

- La soberanía individual (self-sovereignty) aparece asociada a la capacidad de los usuarios de ser dueños del proceso, manteniendo el control sobre el nivel de visibilidad deseado, el almacenamiento y la gestión de la información personal.

\section{Blockchain en educación}

Existe un contraste llamativo entre los avances efectivos (y hasta ahora discretos) de la aplicación de Blockchain en educación, y los enunciados declarativos o aspiracionales, que resultan en algunos casos extremos y ostentosos. De algún modo, el impacto predicado es inversamente proporcional al grado de ejecución real de las propuestas.

En este sentido, los trabajos que describen iniciativas que han logrado una utilización efectiva del Blockchain reconocen con cautela su carácter exploratorio y exponen conclusiones austeras. Otros documentos se refieren a iniciativas reales que se encuentran en etapa de diseño o a meros proyectos hipotéticos. Estas se muestran más pródigas y desprejuiciadas en sus predicciones. Al igual que los artículos o blogs de opinión favorables a Blockchain, describen aplicaciones muy diversificadas y profundas, y abusan de las descripciones hiperbólicas narradas en tiempo verbal futuro o en lenguaje desiderativo. El informe KnowledgeWorks Forecast 4.0, por ejemplo, indica:

«De aquí a 10 años, esperamos ver ecosistemas de enseñanza vibrantes que estén centrados en el alumno, sean equitativos, modulares e interoperables, y resilientes. Este nivel de transformación requerirá que los prestadores educativos norteamericanos innoven con audacia, incluso yendo al límite de reconfigurar algunas de las estructuras subyacentes que soportan el aprendizaje (King et al, 2016, 3).»

No obstante, este mismo informe reconoce el alto nivel de incertidumbre que se cierne sobre la potencialidad del Blockchain en educación. Describe, así, cuatro distintos escenarios en los que pueden identificarse — de menor a mayor- los niveles de incorporación que esta tecnología podría tener en organizaciones y actividades educativas (King et al, 2016, 9).

$\mathrm{Si}$ bien ese ejercicio es teórico, refleja suficientemente la diversidad de direcciones y alcances que ha ido teniendo la aplicación de Blockchain en el contexto de la educación. Los más conocidos son los de la Open University UK, la Universidad Woolf, de Oxford, el MIT Lab en asociación con la Learning Machine Company, la Hoberton School, la Universidad de Nicosia, la Sony Global Education, el BBVA Campus Wallet o la plataforma Tutellus, entre otros.

Del análisis de estas iniciativas y de la literatura disponible, se identifican dos grandes niveles de aplicación de Blockchain en educación: el instrumental o extrínseco y el sustancial o intrínseco.

\subsection{Aplicación en nivel instrumental o extrínseco}

Lo propio de la aplicación en el nivel instrumental reside en que afecta a la actividad educativa como a cualquier otra actividad de servicios. Vale decir que se instrumenta sobre aspectos que no son exclusivos de la actividad educativa. En contraste, la aplicación en el nivel intrínseco genera un impacto directo sobre dimensiones sustantivas de la educación, no reproducibles genéricamente en otras actividades. Son aplicaciones instrumentales:

- El uso de criptomonedas para el pago de matrículas o costos de enseñanza constituye la alternativa más accesible para aquellas organizaciones educativas que deseen incorporar tecnología Blockchain sin avanzar aún en su actividad sustantiva. 
- Smart contract. Lo nuevo del contrato inteligente en su vinculación con Blockchain no es la automatización, sino el hecho de que «las computadoras que ejecutan el contrato son monitoreadas por una red descentralizada» (Casey \& Vigna, 2018). En este sentido, se ejecutan sin necesidad de una tercera parte que actúe como validador o garante (Vander Ark, 2018b).

- La aplicación de contratos inteligentes aspira a generar una disminución de costos y tiempos administrativos al automatizarse gestiones que hoy requieren la intervención de personal. En definitiva, se trata de «reemplazar intermediarios por matemáticas» (Grech et al, 2017). El proyecto de la Universidad Woolf, en Oxford, aspira a capitalizar esta ventaja (Woolf University, 2018).

- Gracias a Blockchain, la acreditación del aprendizaje se realiza a través de una certificación digital que no exige verificación por una tercera parte.

- Mediante el uso de esta tecnología, se podrá habilitar el recurso de la Identidad Soberana Verificada (Verified Sovereign Identities), que otorga al usuario pleno dominio sobre sus credenciales de identificación. Estas credenciales estarán alojadas en su dispositivo personal seguro, y el usuario tendrá la potestad de compartirlas parcial o totalmente con otros sin necesidad de intervención de terceras partes certificantes (Gresch et al, 2017).

- En países que los utilizan, la provisión de vouchers para financiamiento de distintos servicios (incluyendo el educativo) se verá simplificada a partir de la implementación de tarjetas prepagadas o tokens asociados a Blockchain (Grech et al, 2017; Vander Ark, 2018b).

\subsection{Aplicación en el nivel sustancial o intrínseco}

Varias de las aplicaciones anteriormente mencionadas pueden trascender el nivel instrumental de aplicación, adquiriendo un alcance mayor y una modalidad específica para la actividad educativa. A continuación, se mencionarán tres casos en los que puede identificarse esta propiedad.

\subsubsection{Certificación digital mediante Blockchain}

La creación, conservación, emisión y trazabilidad de certificaciones académicas promete transformarse radicalmente a partir de la aplicación de Blockcerts, «un set de herramientas de software y comunicación de código abierto que permite a quienquiera empezar a emitir, compartir y verificar credenciales digitales. Complementariamente a estas herramientas de código abierto, aparecieron las primeras implementaciones comerciales ajustadas a los estándares de los blockcerts, y universidades como MIT empezaron a experimentar con diplomas digitales para algunos estudiantes» (Schmidt, 2017). Las iniciativas de blockcerts del MIT o la Universidad de Nicosia demuestran que se trata de logros factibles y relativamente expandibles a otros sistemas o subsistemas educativos.

Blockcerts consta de cuatro componentes (Bartolomé et al, 2017): El distribuidor o la institución que crea certificados digitales. Los certificados propiamente dichos, ajustados en este caso a los requisitos de la iniciativa Open Badges de la Fundación Mozilla, que contienen un amplio rango de afirmaciones sobre destrezas, logros o características del estudiante, todo registrado en una cadena de bloques; el verificador, esto es, alguien que, sin necesidad de depender del distribuidor, verifica que el certificado no ha sido alterado, que lo ha emitido una determinada institución y que corresponde a un determinado individuo. Una cartera de cada estudiante (wallet) en la que almacena sus certificados compartiéndolos con otros, por ejemplo, con sus empleadores.

Hay quienes imaginan que, en un futuro próximo, la educación en todos sus niveles pueda articularse a través de un sistema de certificados abierto, inmodificable, descentralizado y portable, que otorgue pleno dominio y soberanía al usuario sobre su información y simplifique profundamente la engorrosa tarea de emitir, validar, certificar y compartir credenciales académicas (Vander Ark, 2018b; Chen et al, 2018).

\subsubsection{Contratos inteligentes educativos}

La educación descansa fuertemente en acuerdos explícitos o implícitos. Los planes de estudios escolares o universitarios constituyen responsabilidades asumidas institucionalmente. Los padres y alum- 
nos esperan recibir una determinada formación a cambio del cumplimiento por su parte de compromisos académicos y administrativos específicos. Lo que sucede en el nivel macro, se replica en el nivel micro de las actividades. Los programas de las asignaturas configuran contratos en los que se especifican expectativas de logro para cada una de las partes, material de consulta, modalidades de evaluación, etc.

Si bien hoy el uso de contratos inteligentes en educación se centra en cuestiones meramente instrumentales, algunos sueñan con un sistema educativo que extienda el uso de este tipo de contratos para organizar de manera orgánica las prácticas educativas. Es el caso de la utilización del token como instrumento de cambio no monetario para regular el flujo de actividad dentro de un determinado entorno educativo.

«Un Token es un pedazo de código de programación, en formato de contrato inteligente, que corre sobre una Blockchain. El código de programación describe cómo se comportará dicho Token, y su base de datos simplemente mantiene un registro de cuántos Tokens tiene cada persona. (Arzuaga, 2018).»

Esta modalidad de token, denominada token de uso (utility token), representa básicamente una «promesa de uso futuro en la plataforma para quien lo posee: se paga el consumo del servicio con ese tokensı. De este modo, quien posee un token en un determinado entorno educativo basado en esta tecnología, puede utilizarlo como moneda de intercambio para acceder a una determinada formación. También pueden recibirse tokens como resultado de un proceso educativo. Los tokens pueden acumularse, cual monedas, y el sistema educativo pretende adquirir una dinámica más ágil, abierta y desestructurada, como se comprobará a continuación.

Finalmente, hay quienes llegan al extremo de soñar con extender la utilización de los contratos inteligentes para regular procesos de evaluación. «Blockchain asegura la justicia en la evaluación» aseguran Chen et al - y upuede resolver los problemas de asimetría de información y confianza entre extraños a partir de su descentralización e inmutabilidad» $(2018,8)$.

\subsubsection{Estructuras de docencia y gobernanza descentralizadas}

Refiriéndose a la iniciativa de la Universidad Woolf, Vander Ark no duda en calificarla como un «Airbnb para académicos» (2018a, 2). Esto es así porque, en este modelo, el docente no requiere una pertenencia institucional al modo tradicional. Al configurar su oferta a partir de MOOCs y tutorías virtuales, Woolf University ofrece a cualquier académico — con independencia de su filiación académica y localización - la posibilidad de ser parte del proyecto (Woolf University, 2018). Como puede observarse, la ubicuidad de la educación virtual y los contratos inteligentes, potenciados a través de Blockchain, ofrecen ventajas tanto al alumno como al docente, en este caso habilitando oportunidades profesionales hasta entonces inimaginables.

Pese a todo, el modelo de la Woolf University pretende ser a la vez revolucionario y conservador: «Es nuestra ambición que Woolf configure una revolución sin precedentes en la historia de la universidad. Pero en su esencia, Woolf hace posible la más antigua y venerable forma de educación humana: el aprendizaje directo, personal e individual en el arte de pensar (Woolf University, 2017, 4).»

Esta centralidad en el relacionamiento directo está acompañada de otro supuesto no explicitado: la relevancia del contexto institucional (en este caso, la emulación de criterios propios de la Universidad de Oxford). El peso de la institucionalidad se verifica de diversas maneras, entre las cuales prevalece la práctica de la admisión de alumnos según rendimiento y perfil, la aplicación de modelos de evaluación y acreditación estandarizados y comunes a todos los alumnos y equivalentes a los de la enseñanza presencial, y la selección de docentes sobre la base de criterios institucionales igualmente centralizados.

Las posiciones más entusiastas, sin embargo, imaginan propuestas educativas mucho más descentralizadas y menos institucionales. Junto con la habilitación del token como instrumento de cambio, auguran la configuración de auténticos supermercados de aprendizaje o ed marketplaces (Vander Ark, 2018b, 4) cuya oferta aparece conformada por propuestas formales o no formales, surgidas de fuentes diversas, validadas según estándares académicos mínimos, y regidas por las leyes de la oferta y la demanda.

Las declaraciones del White Paper de la plataforma Tutellus dan una idea bastante lograda acerca de la particular dinámica académica que pretenden configurar este tipo de iniciativas (Tutellus, 2017). $\mathrm{El}$ informe KnowledgeWorks Forecast 4.0 Learning on the Block describe un ecosistema semejante apelando 
a una metáfora. Lo compara con una colonia de hormigas, en la que cada individuo conserva su autonomía respecto de cómo configurar su itinerario de formación, sin perder la interdependencia respecto de las restantes y de la colonia (King et al, 2016).

El itinerario personal no necesariamente exige al alumno insertarse en un ámbito tradicional de formación. Aunque estos ámbitos sigan existiendo, un alumno puede optar por armar un programa personalizado, validando las expectativas de competencias a lograr y las rúbricas de evaluación con expertos que actúan separadamente en el marco de un banco de expertos que se renueva permanentemente. Confrontando la propia trayectoria con la de otros usuarios, tanto los alumnos como los proveedores educativos pueden ir ajustando sus propuestas e intereses.

El sistema permite configurar modularmente el propio trayecto académico con cursos o programas tomados de una diversidad de prestadores. Los procesos administrativos se resuelven a través de contratos inteligentes, y Blockchain resuelve todas las cuestiones de reconocimiento de certificados sobre la base de preacuerdos interinstitucionales plasmados en protocolos que también se enriquecen y renuevan permanentemente.

El mercado laboral también participa en esta red. Los empleadores tienen la posibilidad de monitorear el avance de los alumnos y de incorporar a sus plantillas a quienes alcanzan las competencias requeridas. Pueden también volcar su opinión sobre los graduados y programas, de manera que retroalimentan permanentemente el circuito de enseñanza aprendizaje, lo que permite acortar la distancia entre las demandas laborales y la formación impartida.

Este tipo de proyectos promete convertir en realidad la utopía de Illich de configurar un sistema que democratice la enseñanza y promueva el empoderamiento de estudiantes, rompiendo las asimetrías existentes entre maestro y alumno, y permitiendo la configuración de itinerarios personalizados (Narodowski \& Botta, 2017).

La descentralización del claustro se acompaña de la posibilidad cierta de conformar estructuras de gobernanza no tradicionales. Las universidades de Blockchain rompen con los modelos lineales y tradicionales de gestión, e incorporan figuras tan poco familiares en la academia como la del SelfSovereign Protocol Advisor. Otorgan también un rol protagónico a los directores de Tecnología, que dejan de ser meros actores de soporte para convertirse en configuradores del entorno de enseñanzaaprendizaje y hasta de la arquitectura del sistema y sus componentes. En los casos extremos como el de Tutellus, sorprende el carácter mayoritariamente técnico de los perfiles de conducción (Tutellus, 2017).

\section{Análisis crítico sobre la aplicación de Blockchain en educación}

El análisis comparativo entre las iniciativas y prospectivas de vanguardia en materia de Blockchain aplicado a la educación permite identificar profundas semejanzas, a la vez que marcadas diferencias. Existe un relativo consenso respecto del potencial de Blockchain de transformar la actividad educativa, pero no una imagen nítida y acordada respecto del modo y alcance que habrá de tener dicha transformación.

En los párrafos que siguen se intentará aportar un análisis en profundidad que tenga en cuenta las dimensiones más problemáticas de esta aplicación. El foco de atención estará puesto en el nivel sustancial o intrínseco, ya que la aplicación instrumental merece ser evaluada con una simple ponderación de costos y beneficios, lo que excede las pretensiones de este trabajo.

Se centrará en tres aspectos problemáticos cuyas fronteras no son inmediatamente reconocibles, como habrá de percibirse a continuación:

-Alcances y niveles de la certificación

- Grados de centralización y descentralización, e implicancias

-Personalización, democratización e inclusión a través de Blockchain

\subsection{Alcances y niveles de certificación educativa a través de Blockchain}

Como se ha dicho, la idea básica de Blockchain como «libro de contabilidad distribuido» (DLT: distributed ledger technology) consiste precisamente en reemplazar la mediación de terceras partes certificantes por un sistema distribuido de certificadores anónimos que actúan en red. Blockchain pone el centro de la atención en la idea de confianza (Casey et al, 2018) garantizando altos parámetros de seguridad, confiabilidad, certeza, inmutabilidad y trazabilidad. 
La implementación efectiva de los blockecrts en ámbitos educativos parece convalidar la potencialidad de esta herramienta en lo que respecta a la certificación educativa, y — como se ha vistodespierta la ilusión de avanzar más allá, configurando un modelo interconectado global que revolucione profundamente todo el sistema educativo.

Para poder considerar adecuadamente los distintos alcances de esta posible implementación y sus implicancias, es preciso distinguir previamente los diversos niveles de certificación que operan en el sistema educativo:

- Nivel 1: Acreditación de identidad del usuario. Consiste en verificar la identidad de quien presenta un certificado a su nombre, sea este alumno o docente.

- Nivel 2: Certificación del certificado o diploma. Consiste en verificar la legalidad del documento que acredita una determinada formación de un alumno o docente.

- Nivel 3: Acreditación y certificación de la institución que lo emite. Consiste no solo en reconocer la potestad de la institución para emitir el certificado, lo que determina el grado de validez y alcance del documento emitido, sino también en asegurar la calidad de su servicio sobre la base de estándares predeterminados.

- Nivel 4: Aseguramiento de la calidad de la formación impartida. Aunque suele darse por supuesta a partir del aseguramiento de calidad en el nivel 3, este nivel procura asegurar que el servicio educativo que se reconoce en un certificado o programa haya sido efectivamente impartido con la calidad comprometida.

- Nivel 5: Evaluación y certificación del aprendizaje logrado por el alumno. Consiste en verificar si se ha logrado en el estudiante el nivel de conocimiento o competencia que pretendió asegurar la formación impartida y que el certificado reconoce implícita o explícitamente. Según se ha dicho ya, proyectos como el de Verified Sovereign Identities procuran resolver, mediante tecnología Blockchain, las necesidades vinculadas con el nivel 1. Paralelamente, las iniciativas del tipo blockecerts aseguran la confianza en los procedimientos por los que se emiten, validan, conservan y comparten certificaciones académicas (Schmidt, 2017). Garantizan las necesidades del nivel 2 mediante un sistema de verificación igualmente descentralizado.

Avances semejantes serían de gran provecho para el sistema educativo, y eventualmente para otros sistemas de servicios. Se trata de aportes claramente instrumentales. Las iniciativas exploratorias que más han avanzado en el contexto educativo se concentran en estos dos primeros niveles de la certificación.

Los proyectos más ambiciosos proponen transformar el modelo que opera el sistema en los niveles 3,4 y 5 . Se trata de los ámbitos en que se pone en discusión la configuración del sistema educativo y el rol de las instituciones. En la actualidad, estas deben estar acreditadas como tales por un órgano (generalmente público) con potestad para su reconocimiento en función de un proceso de aseguramiento de la calidad (nivel 3), deben garantizar procesos de formación que se ajusten a lo comprometido en términos de enseñanza (nivel 4), y que logren en los alumnos los aprendizajes deseados (nivel 5).

En la educación formal, los niveles 4 y 5 suelen ser parte de las condiciones que se analizan en el marco del aseguramiento de la calidad de nivel 3. Una institución es reconocida formalmente como tal y se la habilita para operar en la medida en que logra niveles de calidad suficiente en ambos planos, además de otras exigencias. La cuestión de la centralización o descentralización, aspecto central en la discusión de Blockchain, refiere directamente a asuntos de esta índole.

\section{2. ¿Centralización o descentralización?}

Por su misma naturaleza, Blockchain favorece la dinámica de red, lo que determina una inercia más proclive a la articulación entre instituciones que a la custodia de una dinámica institucional centralizada. La idea del ed marketplace resulta funcional al espíritu con que comulgan los promotores de esta tecnología en educación.

Ahora bien, existen dos formas marcadamente diferentes de diseñar este mercado educativo, según se lo conciba como funcionando con un código cerrado dentro de un ámbito o radio limitado (una suerte de «mercado privado») o como operando de manera global, con código abierto y acceso irrestricto. En ambos casos, cabe admitir la participación de una diversidad de instituciones o personas 
que puedan operan desde nodos descentralizados, en el marco de contratos inteligentes y un sistema de certificación ágil, seguro, trazable y no mediado.

\subsubsection{Blockchain de código cerrado}

En este caso, la validez de las certificaciones otorgadas solo alcanza a quienes acuerdan participar del conglomerado y el valor de las mismas fuera de este depende del grado de reconocimiento que tenga la institución o ámbito bajo la cual se nuclean, o del que tenga cada institución miembro.

Pongamos el caso de la BBVA Campus Wallet. Constituye una plataforma basada en Blockchain que articula cursos y talleres presenciales y online, y complementa la formación necesaria para la función profesional. Tiene como objetivo impulsar la formación continua dentro de las organizaciones que forman parte del grupo BBVA. Se compone de más de 7000 recursos que agrupan desde cursos de larga duración hasta la lectura de un artículo o un video. El empleado, con la cantidad de tokens que ha recibido, puede acudir al catálogo y adquirir el programa de formación que desee realizar, tenga relación o no con su actual función. La lógica de la ubicuidad funciona para lo virtual, lo cual potencia la interacción internacional y permite el acceso a mayor cantidad y variedad de contenidos.

Es claro que el valor de la certificación adquirida es intrínseco al grupo BBVA, y se ajusta a los criterios institucionales vigentes. Tiene lugar la dinámica de mercado descentralizado que usualmente acompaña la lógica de Blockchain, pero se trata de un mercado bien delimitado, abierto solo a los miembros del grupo y conformado por una oferta preseleccionada por los responsables de formación, vale decir dominado por una lógica y criterio institucional.

El caso de la Woolf University configura un modelo semejante. Intenta conciliar la lógica de la ubicuidad para alumnos y docentes, y la utilización de contratos inteligentes y tokens, pero preservando - como se ha dicho - la centralidad del encuentro sincrónico (virtual) como modalidad pedagógica preferencial. Tanto en esta elección como en los criterios y estándares de aprobación, selección docente y acreditación, la Woolf University emula el sistema de colleges de la Universidad de Oxford. Los alumnos, que podrán estar radicados en cualquier lugar del mundo, también serán seleccionados en virtud de criterios de excelencia. El Ambrose se posiciona como el primer college de la Woolf University (Woolf University, 2018, 23). Estará conformado por treinta docentes, todos ellos de clase mundial por ser o haber sido profesores de la Universidad de Oxford, lo que garantiza su experiencia en enseñanza tutorial, y su alineación con el criterio institucional.

La expectativa del proyecto es sumar otros cuatro colleges antes del lanzamiento formal de la Universidad, que prevé ofrecer reconocimiento formal externo (nacional e internacional) para sus certificaciones, a diferencia de lo que ocurre en el caso de BBVA. El claustro de los primeros cinco colleges fundacionales deberá reunir un $80 \%$ de miembros con doctorados emitidos por alguna de las 200 universidades que lideran el The Times Higher Education World University Rankings 2017 (Woolf University, 2018, 23). No hay docentes en la Woolf University fuera de los de los colleges. Estos constituyen una entidad digital que aparece en la web de Woolf, y no tienen significancia legal para Woolf (Woolf University, 2018, 24). No deben ser confundidos con lo que «coloquialmente puede denominarse Woolf Universityns. Propiamente, la Woolf University no es otra cosa que la red de Blockchain Woolf (Woolf University, 2018, 24), un emprendimiento o desarrollo tecnológico con sede en Gibraltar, porque «el marco legal está preparado para proveer un entorno regulado y progresivo para la tecnología Blockchain» (Woolf University, 2018).

La plataforma Tutellus pretende constituir un híbrido entre los dos casos anteriores: aspira a ofrecer un conjunto de programas y cursos provenientes de prestadores descentralizados y, en tal sentido, es más abierta que las dos anteriores. El criterio de acceso es también más abierto, en tanto reúne alumnos de cualquier procedencia bajo la única condición de que puedan adquirir tokens (los que se ofrecen gratuitamente en primera instancia). Lo cierto es que la plataforma constituye un emprendimiento privado cuyas normas de funcionamiento, criterios de aplicación, selección docente, gobernanza de tokens, etc., se definen unilateralmente desde los desarrolladores. La validez de las certificaciones es intrínseca a Tutellus, al menos en tanto sus componentes formativos o la institución cuenten con reconocimiento externo dentro del sistema formal de enseñanza.

Las tres iniciativas mencionadas promueven ventajas comparativas que están más asociadas a la consolidación de la enseñanza virtual que a la aplicación de la tecnología Blockchain. En los tres casos se pone en evidencia una descentralización relativa. No desaparece la centralización ni la institucionalidad, aun cuando en algún caso estemos en presencia de un nuevo paradigma de institucionalidad 
y centralización más afín a la tipología de las redes que a las organizaciones tradicionales de enseñanza. En este paradigma, las instituciones educativas son "plataformas» tecnológicas que se constituyen como emprendimientos privados.

\subsubsection{Blockchain de código abierto}

Ahora bien, las prospectivas más extremas refieren a un sistema absolutamente descentralizado, lo cual exige una implementación de Blockchain bajo el modo de open source o código abierto. Estas prospectivas están alineadas con la enfática advertencia que explicita la Unión Europea a través de su informe «Blockchain en educación», de 2017:

«Solo las implementaciones de Blockchain totalmente abiertas pueden alcanzar las metas y promesas reales de Blockchain en educación. Por esto entendemos a las soluciones que incluyan entre sus componentes esenciales: a) la soberanía del destinatario; b) la independencia del prestador y c) una verificación descentralizada. Si no se alcanzan todas estas condiciones, el uso de Blockchain constituirá en una pérdida de tiempo y recursos para todas las partes interesadas.» (Grech et al, 2017, 101).

En esta misma línea, Vander Ark no duda en afirmar que «Blockchain va a eliminar los certificados impresos, automatizar la adquisición, reconocimiento y transferencia de créditos, incrementar la apropiación y el control de los propios datos por parte del dueño, reducir los costos y riesgos institucionales, solo si se adoptan estándares abiertos» (Vander Ark, 2017). Esto no sucede en ninguno de los casos anteriormente mencionados, lo que alerta sobre la posibilidad cierta de que se trate de proyectos que podrían generar nuevos modos de dependencia de terceras partes (Grech et al, 2017).

Los proyectos educativos de código abierto basados en Blockchain existen solo en declaraciones o aspiraciones formalizadas. Su universalización y adopción global se enfrenta a un primer desafío de orden práctico, referido a la transferibilidad y acumulabilidad del conocimiento. La transferibilidad implica interoperabilidad de la certificación. Y la interoperabilidad supone un sistema de acreditación transinstitucional tal que permita a escuelas, universidades, instituciones formativas formales y no formales de todas las comunidades, regiones, países y culturas, homologar sus certificaciones. En otras palabras, se trata de un sistema de homologación universal que resuelva las necesidades de validación transnacional, intranacional, intersectorial e interinstitucional de certificaciones (Bartolomé et al, 2017).

Ahora bien, los proyectos basados en Blockchain aspiran a resolver la homologación sin mediaciones de terceras partes, a través de cómputos algorítmicos, y no exclusivamente para el nivel 1 y 2 de la certificación, sino también para los restantes tres niveles de certificación. Esto genera desafíos sobreañadidos:

-De nivel 3: ¿cómo se garantiza la interoperabilidad entre certificados emitidos por una diversidad de prestadores si algunos de ellos cuentan con niveles de acreditación y certificación institucional y otros no? En otras palabras, ¿cómo se reconocen en el marco del sistema de homologación los certificados de instituciones formales y no formales?

-De nivel 4: ¿cómo asegura el sistema que la formación certificada haya sido efectivamente impartida? En términos prácticos, este problema constituye una derivada del anterior, en tanto que las instituciones acreditadas para la enseñanza lo son, entre otras cosas, porque aseguran el servicio que comprometen.

-De nivel 5: ¿cómo asegura el sistema Blockchain que la formación impartida y certificada es efectivamente tal? ¿Qué valor tendrán las opiniones de los empleadores en tanto potenciales certificadores de formación adquirida por los alumnos?

En rigor, estos tres desafíos existen para el sistema educativo desde siempre. El modo en que el sistema tradicional procura resolverlos implica, precisamente, la participación de terceras partes validantes. En tanto Blockchain aspira a reemplazarlas con operaciones algorítmicas matemáticas, el desafío de la homologación universal está precedido por el desafío de la construcción de un sistema de estandarización objetivo y objetivable en términos matemáticos que elimine cualquier criterio interpretativo ejercido por un validador o certificador externo en cualesquiera de los mencionados niveles. Una estandarización total del proceso educativo implicaría la reducción de los procesos educativos a dimensiones transables/homologables, vale decir, susceptibles de verificación computarizable (Chen et al, 2018). 
En su aspiración extrema, esto podría afectar a cuestiones esenciales del acto educativo. Por lo pronto, se requerirá del docente la minimización de cualquier posibilidad de sesgo interpretativo en la evaluación. A fin de asegurar la plena homologabilidad, es conveniente que este aplique modalidades de evaluación «computarizables», lo que conlleva el riesgo de confundir educación con evaluación, y evaluación con certificación (Bartolomé et al, 2017).

En segundo término, la eliminación de terceras partes validantes, implica la idea de igualación (en nombre de la «democratización») de los docentes proveedores de formación. Un mercado educativo abierto debiera permitir la participación general de quien quiera ofrecer una propuesta de formación, con independencia de la pertenencia institucional que ostente y la validación que otorgue dicha institución sobre su rol y jerarquía.

Surge inevitablemente la pregunta respecto de quién valida y certifica la calidad del prestador, rol atribuible a las instituciones en el actual sistema. Tutellus introduce una novedad llamativa a efectos de resolver esta necesidad de garantizar calidad. Homologa la dinámica de la plataforma con la de las redes sociales, jerarquizando la importancia de la opinión del usuario e incorporando el rol del influencer a efectos de movilizar preferencias (Tutellus, 2017). Reemplaza, así, el rol validador y certificador de una institución educativa por la valoración que surge de la dinámica de interacciones de las redes. Así como el influencer es premiado con tokens por su tarea de persuasión, el docente es reconocido y valorado por el nivel de satisfacción que alcanza en sus cursos, medido a partir de las interacciones con los alumnos e influencers en el marco de la red.

Como se comprenderá, el reemplazo del rol institucional por el de las iteraciones en redes sociales implica una transformación importante en la lógica del sistema educativo y de la relación docentealumno, de naturaleza jerárquica y asimétrica, e implica una redefinición importante del rol de las instituciones educativas. Cabe preguntarse sobre las implicancias de un cambio semejante en términos cualitativos del sistema educativo.

Esta relativización del rol de las instituciones abre interrogantes respecto del rol de los gobiernos u organismos de gobiernos responsables de velar por la calidad del sistema educativo. En el sistema actual, a ellos les corresponde acreditar instituciones de enseñanza y validar criterios y estándares generales para el sistema educativo.

Como cabe esperar, el informe Blockchain en educación de la Unión Europea enfatiza que el rol de los gobiernos no debe ser reemplazado y que los gobiernos deben conservar esta potestad. Advierte, sin embargo, que el proyecto Blockchain en educación exigirá el logro de un acuerdo suficientemente amplio entre gobiernos e instituciones que asegure la factibilidad de la implementación y la inmutabilidad de las transacciones a través de Blockchain. También remarca la necesidad de garantizar la trazabilidad de la formación multinstitucional impartida a través del reconocimiento y transferencia automática de créditos, lo que se cristaliza en la existencia de un "pasaporte del aprendizaje de toda la vida» (Grech et al, 2017, 96-98). Además de su complejidad operativa, la implementación supone reconsiderar el rol de la educación no formal e informal y la modalidad de articulación con la educación formal. La Unión Europea augura, por tanto, un tránsito largo y complejo para el logro de todos estos cometidos.

\subsection{Personalización, democratización e inclusión a través de Blockchain}

Como es sabido, el desafío de la educación personalizada quedó desde el inicio constreñido por las dinámicas de un sistema forjado en la modernidad, preparado para acompañar grupos relativamente homogéneos, signado por normativas atentas a la uniformización antes que a la individualización, y poco dócil al acompañamiento de trayectorias customizadas o a la medida de cada alumno.

Según se ha dicho, la irrupción de la enseñanza virtual ofreció alternativas para desafiar algunas de estas limitaciones. Blockchain promete potenciar aún más esta impronta de personalización, bajo la premisa de avanzar en tres direcciones necesarias y complementarias.

En primer lugar, y en tanto se instrumente una homologación global y el acceso a un sistema de certificación transinstitucional y transnacional, lograrán superarse las barreras que actualmente condicionan el tránsito desde y hacia la educación no formal o informal. Cumplida esta condición, quedará habilitada la posibilidad de instaurar un sistema global de educación basado en la idea de un «mercado abierto» que rompa con la noción e instrumentación de un currículum preestablecido. 
Resulta algo paradójico que el requisito de la estandarización y homologación universal pretenda constituirse en una garantía de personalización. Es cierto que la necesidad de estandarización matemática está en la base de la lógica de la customización en el orden tecnológico. Indudablemente, es deseable avanzar en esta dirección para los niveles más instrumentales de la educación.

Cabe preguntarse, sin embargo, en qué medida esta lógica debe ser aplicada a todo el sistema educativo. ¿Qué espacio habría en un contexto semejante para lo no considerado en los estándares, lo no protocolizado? ¿Acaso será reconocible para el alumno? ¿Cuáles serían los contenidos estrictamente homologables y certificables y quién habrá de definirlos?

Una segunda cuestión vinculada con lo anterior tiene que ver con la eventual eliminación del currículum preestablecido y la necesaria (o innecesaria) existencia de «mínimos formativos». El sistema formal de educación es destinatario de demandas y debe actuar como garante para responder a un sinnúmero de expectativas. En el nivel escolar, estas refieren a la necesidad de inserción en el mundo del trabajo, a la formación de una ciudadanía moralmente íntegra y apta para desenvolverse en un mundo globalizado, a la creación de disposiciones favorables a la inclusión y respetuosas de las diferencias, a la promoción integral de la persona, al compromiso con los derechos humanos y la preservación del ambiente, entre otros requerimientos. En los niveles superiores de formación, el cumplimiento de los mínimos formativos adquiere un grado de especialización y exigencia que es proporcional a la criticidad de la formación impartida (ej.: la formación de médicos). Ahora bien, ¿acaso la demanda de personalización radical implica el abandono de la formación en torno a estos mínimos?

Si no fuera así, ¿̇a quién corresponde definir y custodiar el cumplimiento de los mismos? ¿Cómo se concilia la lógica de la personalización radical con esta necesidad de homogeneización universal que opera en la lógica del sistema? Los modelos analizados no parecen responder a estos interrogantes.

En tercer y último lugar, es preciso considerar la cuestión de la accesibilidad. Esta compromete, entre otras cosas, la dimensión de las posibilidades de acceso a la tecnología, así como el análisis de los costos individuales o globales.

La mayoría de las prospectivas mencionan el factor de la disminución de costos como corolario inherente a la automatización de procedimientos. Algunos van más allá y destacan la posibilidad de que, a partir de la implementación de Blockchain en educación, el alumno no solo pierda menos dinero sino también pueda ganar dinero (learning is earning) (Chen et al, 2018). El learning by earning pretende incorporar un elemento de motivación extrínseca atractivo que, en una dinámica descentralizada como la que asume Blockchain, asume que «los miembros de la comunidad sean los que determinen las recompensas a obtener, en base al esfuerzo aportado y la calidad conseguida» (Tutellus, 2018, 12). De este modo, Blockchain promete ser una tecnología capaz de generar inclusión y disminuir la incidencia de la pobreza (Tutellus, 2016).

Estas valoraciones, ciertamente insuficientes y en algún sentido discutibles, contrastan con los análisis forjados dentro de la comunidad misma de los usuarios y desarrolladores de Blockchain, que alertan sobre el enorme consumo de energía que compromete su funcionamiento. En un portal para especialistas en Blockchain (Blockgeeks) la problemática apareció explicada ya en 2016. La naturaleza competitiva de la minería de datos ha generado que el nivel de complejidad ascienda y, por tanto, se incrementen el tiempo y los costos requeridos para que un minero añada un bloque a la cadena.

«En el consenso distribuido Proof of Work, los mineros necesitan mucha energía. Una transacción de Bitcoin requirió la misma cantidad de electricidad que la necesaria para alimentar 1,57 familias norteamericanas por día (datos de 2015). Y estos costos energéticos son pagados con moneda analógica, ejerciendo una presión constante sobre el valor de la moneda digital. En un estudio reciente, expertos aseguraron que las transacciones de Bitcoin podrían consumir para 2020 tanta electricidad como la que consume Dinamarca.» (Rosic, 2017).

De allí que algunos desarrolladores estén implementando otro protocolo de validación o consenso llamado Proof of Stake que abre, a su vez, interrogantes respecto del grado de descentralización finalmente logrado en la implementación de las validaciones, entre otros aspectos (Rosic, A., 2018). Como podrá verse, la prerrogativa de inclusión del Blockchain parece constituir un mito antes que una realidad si se considera el fenómeno en su dimensión global.

El problema se agrava cuando se analiza en perspectiva regional. La mayoría de los modelos basados en Blockchain parten del supuesto de la equiparabilidad entre lo presencial y lo virtual. Más 
allá del análisis crítico que podría hacerse respecto de dicho supuesto desde un punto de vista pedagógico, su aplicabilidad remite a determinadas condiciones socioeconómicas que no necesariamente son asequibles para gran parte de la población latinoamericana. Nos referimos a condiciones de acceso a la tecnología, la conectividad o, incluso, más radicales, como la provisión de servicio eléctrico.

\section{Conclusiones}

«La relación de las personas con las instituciones está cambiando, y las nuevas tecnologías están propiciando este cambio», afirma el informe KnowledgeWorks Forecast 4.0 (King et al, 2016, 22).

Como puede observarse, la magnitud de la transformación que algunos autores atribuyen a Blockchain dista de corresponderse con las implementaciones efectivas ya iniciadas. No obstante, la lectura de las prospectivas más importantes y de los documentos alusivos a su implementación futura ofrece una imagen ambivalente respecto del impacto y conveniencia de una aplicación indiscriminada y global.

Por un lado, los primeros proyectos efectivamente implementados basados en Blockchain se enmarcan claramente sobre la dinámica tradicional del sistema educativo, sea en ámbitos formales, sea en ámbitos no formales, y sus alcances aparecen circunscriptos a cuestiones de certificación de identidad y de diplomas.

Los proyectos más audaces proponen introducir un cambio de paradigma en tanto y en cuanto reemplazan o eventualmente subsumen la institucionalidad de la escuela, el instituto o la universidad (todas ellas constituidas según el patrón arquetípico de «comunidad de alumnos y docentes») en la lógica de las plataformas tecnológicas multilaterales.

Estos desarrollos educativos se basan en plataformas de código cerrado que utilizan la tecnología Blockchain sin trasladar sus principales ventajas o atributos a la dinámica institucional. En definitiva, configuran emprendimientos privados con un grado de descentralización relativa. El grado de reconocimiento formal de las certificaciones queda asociado al tipo de formación impartida (formal o no formal), por un lado, y al contexto legal vigente en el país de radicación del proyecto, por otro. A su vez, la nueva institucionalidad de la plataforma supone la creación de figuras jurídicas igualmente ajenas a la dinámica educativa tradicional.

Es preciso destacar, a su vez, que la tecnología Blockchain no introduce ninguna novedad en la dinámica de la enseñanza-aprendizaje. Las propuestas didácticas de plataformas como BBVA o Tutellus conllevan todas las fortalezas y debilidades propias de los portales de capacitación tradicionales. Acaso Woolf University ofrece algún grado de innovación al pretender reemplazar la tutoría presencial oxoniense con tutorías virtuales uno-a-uno. Pero en ninguno de estos casos, u otros que podrían mencionarse, Blockchain interviene promoviendo una mejora de la didáctica o una contribución para el logro de mayor o mejor aprendizaje. Sus innovaciones ocurren en la periferia del acto propiamente educativo.

Las prospectivas más extremas aspiran a redefinir dimensiones propias de la arquitectura del sistema educativo, en una dinámica multinstitucional que intentará diluir las fronteras entre la educación formal y la no formal, promoviendo un mercado educativo transnacional. No es posible dimensionar el impacto pedagógico de esta eventual transformación, dado que no existen proyectos de código abierto que hayan logrado reproducir las ventajas de Blockchain en la dinámica educativa.

Este tipo de proyectos aún no cuentan con condiciones de realización (que no constituyen necesariamente logros tecnológicos), ni necesariamente parecen aportar mejoras respecto de la actual dinámica del sistema educativo. Si bien, como se ha visto, no está descartada la posibilidad hipotética de lograr un acuerdo intergubernamental que determine estándares de homologación globales en los niveles de certificación más básicos, y que se configure un entorno normativo acorde a la implementación de iniciativas basadas en Blockchain, la factibilidad de este desarrollo merece ser puesta en duda.

Asimismo, debe ser cuidadosamente analizada la posibilidad de reemplazar la mediación de los organismos gubernamentales por otra instancia de validación, trátese de usuarios actuando según la dinámica de una red social, o de cualquier otro mecanismo que resuelva las necesidades de validación.

Todo lo dicho invita a pensar — al menos en una primera etapa exploratoria - en la alternativa de un sistema mixto que complete y potencie las fortalezas del actual sistema educativo formal con las ventajas que podría traer aparejada la implementación de una plataforma pública de código abierto basada en Blockchain. 


\section{Referencias}

Arzuaga, G. (2018). Criptomonedas, Madrid: Conecta. Penguin Random House.

Bartolomé, A. R., Bellver, C., Castañeda, L., Adell, J. (2017). «Blockchain en educación: introducción y crítica al estado de la cuestión». EDUTEC, Revista Electrónica de Tecnología Educativa, 61. Recuperado el 14 de mayo de 2018 de: http://www.edutec.es/revista/index.php/edutec-e/article/view/915. doi: doi.org/10.21556/edutec.2017.61.915.

Burbules, N. (2009). "Meanings of ubiquitous learning”. In Cope, B. \& Kalantzis, M. (eds.); Ubiquitous Learning. Urbana II: University of Illinois Press, pp. 15-20.

Casey, M. \& Vigna, P. (2018), “In Blockchain we trust”. MIT Technology Review, May/Jun 2018 Issue. Recuperado el 10 de abril de 2019 de https://www.technologyreview.com/s/610781/in-blockchain-we-trust/.

Chen, G., Xu, B., Lu, M. \& Chen, M. S. (2018). "Exploring Blockchain technology and its potential applications for education". Smart Learning Environments, December 2018, 5: 1. doi: doi.org/10.1186/s40561-017-0050-x.

Clark, D. (2016). "10 ways Blockchain could be used in education”. OEB Insights. Recuperado el 23 de diciembre de 2017 de https://oeb.global/oeb-insights/10-ways-blockchain-could-be-usedin-education/.

Franceschin, T. (2017), «Los Bootcamps: una modalidad de aprendizaje en crecimiento». Edu4me. Recuperado el 15 de febrero de 2017 de http://edu4.me/los-bootcamps-una-modalidad-deaprendizaje-en-crecimiento/.

Grech, A. \& Camillery, A. (2017), "Blockchain in education”. JRC Science for Policy Report , Luxembourg: Publications Office of the European Union, 132 S. Recuperado el 26 de marzo de 2018 de http://publications.jrc.ec.europa.eu/repostory/bitstream/JRC108255/jrc108255_blockchain_in_education(1).pdf.

King, K., Prince, K \& Swanson, J. (2016), "Learning on the Block: Could Smart Transactional Models Help Power Personalized Learning?”. KnowledgeWorks Forecast 4.0. Recuperado el 2 de mayo de 2017 de https://knowledgeworks.org/resources/blockchain-smart-transactional-models-2/.

Narodowski, M. y Botta, M (2017); «La mayor disrupción posible en la historia de la pedagogía moderna: Ivan Illich». Pedagogía y saberes. Buenos Aires: Universidad Pedagógica Nacional, Núm. 46; pp.45-54. doi: doi.org/10.17227/01212494.46pys45.54.

Oakeshott, M. (2000). «El racionalismo en política». En El racionalismo en la política y otros ensayos. México, FCE, 21-53.

Rosic, A. (2017), "Proof of Work vs Proof of Stake: Basic Mining Guide". Blockgeeks. Recuperado el 14 de abril de 2018 de https://blockgeeks.com/guides/proof-of-work-vs-proof-of-stake/.

Rosic, A. (2018), "Decentralization has become Synonymous with Blockchain. But is it the truth?". Blockgeeks. Recuperado el 14 de abril de 2018 de https://blockgeeks.com/decentralization-hasbecome-synonymous-with-blockchain-but-is-it-the-truth/.

Schmidt, P. (2017), "Credentials, Reputation, and the Blockchain", EducauseReview. Recuperado el 14 de abril de 2018 de https://er.educause.edu/articles/2017/4/credentials-reputation-and-the-blockchain.

Tutellus. Bienvenido a la educación del futuro. White Paper. Julio 2018, ver. 3.27. Recuperado el 17 de abril de 2018 de https://lib.tutellus.com/ico/pdf/tutellus.io whitepaper v4.0 en.pdf.

Vander Ark, T. (2017), "How Blockchain Will Transform Credentialing (and Education)", Getting Smart, December 21, Recuperado el 24 de abril de 2018 de http://www.gettingsmart.com/2017/12/blockchain-will-transform-credentialing-education/.

Vander Ark, T. (2018a), "Imagining A Blockchain University", Forbes, Jun 13. Recuperado el 14 de abril de 2018 de https://www.forbes.com/sites/tomvanderark/2018/06/13/woolf-building-thefirst-blockchain-university/\#1daa50b05ae5.

Vander Ark, T. (2018b), "20 ways Blockchain Will Transform (Okay, May Improve) Education", Forbes, Aug. 20. Recuperado el 14 de abril de 2018 de https://www.forbes.com/sites/tomvanderark/2018/08/20/26-ways-blockchain-will-transform-ok-may-improve-education/\#70a11684ac91. 
Watters, A. (2016). "The ideology of the Blockchain (for Education). HackEducation, April 14th, 2016. Recuperado el 24 de abril de 2018 de http://hackeducation.com/2016/04/14/blockchain-ideology.

Woolf University (2018), White Paper. Building the First Blockchain University, Aug. 2018. Recuperado el 28 de diciembre de 2018 de https://woolf.university/assets/doc/whitepaper.pdf. 